\title{
MicroRNA-200c inhibits the metastasis of non-small cell lung cancer cells by targeting ZEB2, an epithelial-mesenchymal transition regulator
}

\author{
AIHONG JIAO ${ }^{1,2^{*}}$, MINGHUA SUI $^{2 *}$, LIANGMING ZHANG ${ }^{2}$, PING SUN $^{2}$, \\ DONGMEI GENG ${ }^{2}$, WEIWEI ZHANG ${ }^{2}$, XIUWEN WANG ${ }^{1}$ and JUNXIA LI ${ }^{2}$
}

${ }^{1}$ Department of Chemotherapy, Cancer Center, Qilu Hospital of Shandong University, Jinan, Shandong 250012;

${ }^{2}$ Department of Chemotherapy, Yuhuangding Hospital, Yantai, Shandong 264000, P.R. China

Received March 31, 2015; Accepted January 25, 2016

DOI: $10.3892 / \mathrm{mmr} .2016 .4901$

\begin{abstract}
MicroRNAs (miRs) have been demonstrated to regulate various biological processes in human cancer, including non-small cell lung cancer (NSCLC). However, little evidence has been provided regarding the exact role of miR-200c in mediating the malignant progression of NSCLC, as well as the underlying mechanism. The present study aimed to investigate the putative role of miR-200c in the progression of NSCLC. The expression levels of miR-200c were significantly reduced in NSCLC cell lines compared with in normal lung epithelial cells, as determined by reverse transcription-quantitative polymerase chain reaction. Overexpression of miR-200c significantly suppressed cell migration and invasion of A549 NSCLC cells. Results of a luciferase reporter assay further identified zinc finger E-box-binding homeobox 2 (ZEB2) as a direct target gene of $\mathrm{miR}-200 \mathrm{c}$, and the expression of ZEB2 was shown to be suppressed in A549 cells overexpressing miR-200c. Furthermore, small interfering RNA-mediated inhibition of ZEB2 suppressed the migration and invasion of A549 cells. In addition, since ZEB2 is an epithelial-mesenchymal transition (EMT) regulator, the role of miR-200c in the regulation of EMT in NSCLC cells was further examined. Results of a western blot analysis indicated
\end{abstract}

Correspondence to: Professor Xiuwen Wang, Department of Chemotherapy, Cancer Center, Qilu Hospital of Shandong University, 107 Wenhua West Road, Jinan, Shandong 250012, P.R. China

E-mail: qiluwangxiuwen@163.com

Professor Junxia Li, Department of Chemotherapy, Yuhuangding Hospital, 20 Yuhuangding East Road, Yantai, Shandong 264000, P.R. China

E-mail: 13356997292@163.com

"Contributed equally

Key words: non-small cell lung cancer, microRNA, zinc finger E-box-binding homeobox 2, metastasis, epithelial-mesenchymal transition that overexpression of miR-200c upregulated E-cadherin, and downregulated $\mathrm{N}$-cadherin and vimentin expression in A549 cells, thus suggesting that EMT was suppressed. Based on these results, the present study suggested that miR-200c was able to inhibit the metastasis of NSCLC cells by targeting ZEB2. Therefore, miR-200c may be considered as a potential candidate for the treatment of NSCLC.

\section{Introduction}

Lung cancer is the leading cause of cancer-associated mortality worldwide, the incidence of which is increasing (1). Non-small cell lung carcinoma (NSCLC) accounts for the majority of lung cancer cases. The majority of cases of NSCLC are adenocarcinoma, squamous cell carcinoma, and large cell carcinoma (2). Despite great efforts being made regarding the treatment of NSCLC, and recent improvements in surgery, radiotherapy and chemotherapy, the overall survival rate of patients with NSCLC remains poor $(1,3)$. Therefore, a more effective therapeutic strategy is urgently required.

MicroRNAs (miRs) are short non-coding RNAs, which can inhibit protein translation, or degrade mRNA by binding to seed sequences within the 3'-untranslational region (UTR) of target mRNAs (4). It has previously been demonstrated that miRs are important regulators in various biological processes, including cell proliferation, apoptosis, survival, differentiation and motility (5). Furthermore, several targets of miRs have been reported to be highly associated with tumorigenesis and cancer metastasis $(6,7)$. Recent studies have suggested that aberrant expression of miR-200c is associated with the development and progression of NSCLC. Ceppi et al (8) reported that loss of miR-200c expression induces an aggressive, invasive and chemoresistant phenotype in NSCLC. Li et al (9) demonstrated that miR-200c is able to inhibit the invasion and metastasis of NSCLC by directly targeting ubiquitin specific peptidase 25. Furthermore, miR-200c may increase the radiosensitivity of A549 NSCLC cells by targeting the vascular endothelial growth factor (VEGF)-VEGF receptor 2 pathway (10). Since one miR has several targets, whether other targets of miR-200c are associated with miR-200c-mdiated NSCLC malignant phenotypes remains to be elucidated. 
Zinc finger E-box-binding homeobox 2 (ZEB2) is a member of the zinc finger homeodomain 1 family of 2-handed zinc finger/homeodomain proteins. It is located in the nucleus and functions as a DNA-binding transcriptional suppressor (11). ZEB2 has been reported to suppress the expression of E-cadherin, and promote the transcription of vimentin and $\mathrm{N}$-cadherin $(11,12)$, thus suggesting that ZEB2 acts as a promoter of epithelial-mesenchymal transition (EMT). Furthermore, ZEB2 has been reported to be involved in the migration, invasion and EMT of NSCLC cells (13). However, the association between miR-200c and ZEB2 in NSCLC has yet to be studied.

The present study aimed to explore the potential role, as well as the regulatory mechanism, of miR-200c in mediating the metastasis of NSCLC in vitro.

\section{Materials and methods}

Cell lines. NSCLC cell lines: A549, H358, H460 and H1229, and the BEAS-2B normal human lung epithelial cell line were obtained from the Chinese Academy of Sciences (Shanghai, China). All cell lines were cultured in Dulbecco's modified Eagle's medium (DMEM; Thermo Fisher Scientific, Inc., Waltham, MA, USA) supplemented with $10 \%$ fetal bovine serum (FBS; Thermo Fisher Scientific, Inc.), 100 IU/ml penicillin and $100 \mathrm{IU} / \mathrm{ml}$ streptomycin (both purchased from Thermo Fisher Scientific, Inc.) at $37^{\circ} \mathrm{C}$ in a humidified atmosphere containing $5 \% \mathrm{CO}_{2}$.

Reverse transcription-quantitative polymerase chain reaction (RT-qPCR) assay. Total RNA was extracted from the cells using TRIzol ${ }^{\circledR}$ reagent (Invitrogen; Thermo Fisher Scientific, Inc.). For the detection of miR-200c expression, a miRNA Reverse Transcription kit (Thermo Fisher Scientific, Inc.) was used to convert RNA into cDNA, according to the manufacturer's protocol. qPCR was performed using a miRNA Q-PCR Detection Kit (Genecopoeia, Rockville, MD, USA) on an Applied Biosystems 7500 thermocycler (Applied Biosystems; Thermo Fisher Scientific, Inc.). The final reaction volume was $20 \mu \mathrm{l}$, including $1 \mu \mathrm{l}$ cDNA, $10 \mu \mathrm{l}$ PCR Master Mix (GeneCopoeia), $2 \mu \mathrm{l}$ primer and $7 \mu \mathrm{l} \mathrm{H}_{2} \mathrm{O}$. The PCR conditions were as follows: $95^{\circ} \mathrm{C}$ for $5 \mathrm{~min}$, and 40 cycles of denaturation at $95^{\circ} \mathrm{C}$ for $15 \mathrm{sec}$ and annealing/elongation at $60^{\circ} \mathrm{C}$ for $30 \mathrm{sec}$. Primers were purchased from Sangon Biotech Co., Ltd. (Shanghai, China) and the sequences were as follows: miR-200x Forward, 5'-CTTAAA GCCCCTTCGTCTCC-3' and reverse, 5'-AGGGGTGAA GGTCAGAGGTT-3'; and U6 forward, 5'-TGCGGGTGC TCGCTTCGGCAGC-3' and reverse, 5'-CCAGTGCAGGGT CCGAGGT-3'. U6 gene was used as an internal reference. The relative expression levels were analyzed using the $2^{-\Delta \Delta \mathrm{Cq}}$ method (14).

Transfection. Lipofectamine ${ }^{\circledR} 2000$ (Invitrogen; Thermo Fisher Scientific, Inc.) was used to conduct transfection of the cells, according to the manufacturer's protocol. miR-200c mimic, scramble mimic, miR-200c inhibitor (Shanghai Genechem Co., Ltd., Shanghai, China) ZEB2 small interfering (si)RNA or negative control siRNA and Lipofectamine ${ }^{\circledR} 2000$ were diluted in serum-free DMEM. The diluted
Lipofectamine 2000 was added to the diluted miR mimic or siRNA $(100 \mathrm{nM})$. Following a $20 \mathrm{~min}$ incubation at room temperature, the mixture was added to a cell suspension $(5,000,000$ cells $/ \mathrm{ml})$. Following a $10 \mathrm{~h}$ incubation at $37^{\circ} \mathrm{C}$ in an atmosphere containing $5 \% \mathrm{CO}_{2}$, the medium was replaced with DMEM supplemented with $10 \%$ FBS.

Western blot analysis. Cells were lysed with ice-cold lysis buffer [50 mM Tris-HCl, pH 6.8; $100 \mathrm{mM} \mathrm{2-ME,} \mathrm{2 \% w/v}$ sodium dodecyl sulfate (SDS), $10 \%$ glycerol]. Proteins $(50 \mu \mathrm{g})$ were separated by $10 \%$ SDS-polyacrylamide gel electrophoresis, and were then transferred onto a polyvinylidene difluoride (PVDF) membrane (GE Healthcare Life Sciences, Chalfont, UK). The PVDF membrane was then blocked with phosphate-buffered saline (Thermo Fisher Scientific, Inc.) containing $5 \%$ milk overnight at $4^{\circ} \mathrm{C}$, and was incubated with rabbit anti-ZEB2 polyclonal antibody (1:200; ab138222, Abcam, Cambridge, MA, USA), rabbit anti-E-cadherin monoclonal antibody (1:200; ab15148; Abcam), rabbit anti-vimentin monoclonal antibody $(1: 100$; ab92547; Abcam), rabbitanti-N-cadherin polyclonal antibody (1:200; ab12221; Abcam), and rabbit anti-glyceraldehyde 3-phosphate dehydrogenase (GAPDH) polyclonal antibodies (1:200; ab9485; Abcam) at room temperature for $3 \mathrm{~h}$. Subsequently, the membrane was incubated with horseradish peroxidase-conjugated goat anti-rabbit secondary antibody (1:10,000; ab150077; Abcam) at room temperature for $1 \mathrm{~h}$. The signals on the PVDF membrane were detected using the Super Signal West Pico Chemiluminescent Substrate kit (Pierce Biotechnology, Inc., Rockford, IL, USA), according to the manufacturer's protocol. Protein concentration was quantified using a BCA Protein Assay Kit (Thermo Fisher Scientific, Inc.) according to the manufacturer's instruction. The relative protein expression levels were analyzed using Image-Pro Plus software 6.0 (National Institutes of Health, Bethesda, MD, USA), and are presented as the density ratio vs. GAPDH.

Cell migration assay. A549 NSCLC cells were cultured to full confluence in 6-well plates, and wounds (width, $1 \mathrm{~mm}$ ) were created using a plastic scriber. Cells were washed and incubated in DMEM containing $10 \% \mathrm{FBS}$ at $37^{\circ} \mathrm{C}$ in an atmosphere containing $5 \% \mathrm{CO}_{2}$ for $48 \mathrm{~h}$. Subsequently, the cells were fixed with methanol and observed using a microscope (CX31; Olympus Corporation, Tokyo, Japan).

Cell invasion assay. Cell invasion assay was performed using Transwell chambers pre-coated with Matrigel (BD Biosciences, Franklin Lakes, NJ, USA). An A549 cell suspension containing $5 \times 10^{5}$ cells $/ \mathrm{ml}$ was prepared in serum-free DMEM, and $300 \mu \mathrm{l}$ cell suspension was added to the upper chamber. A total of $500 \mu$ l DMEM supplemented with $10 \%$ FBS was added to the lower chamber. Following a $24 \mathrm{~h}$ incubation at $37^{\circ} \mathrm{C}$ in an atmosphere containing $5 \% \mathrm{CO}_{2}$, the A549 cells that did not invade through the membrane were removed from the chamber with a cotton-tipped swab. The upper chamber was then fixed in $90 \%$ alcohol and stained with $0.1 \%$ crystal violet. A549 cells that had invaded through the membrane were observed under an inverted microscope (CX31; Olympus Corporation). 


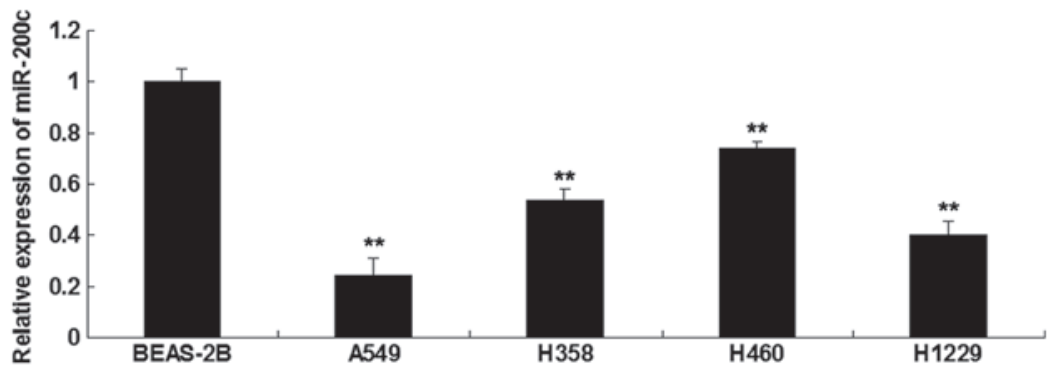

Figure 1. Reverse transcription-quantitative polymerase chain reaction was conducted to detect the expression levels of microRNA (miR)-200c in non-small cell lung cancer cell lines: A549, H358, H460, and H1229, and in the BEAS-2B normal human lung epithelial cell line, which was used as a control. Data are presented as the mean \pm standard deviation. ${ }^{* *} \mathrm{P}<0.05$ vs. the control cells.

Luciferase reporter gene assay. Total cDNA from A549 cells was used to amplify the 3'UTR of ZEB2 using PCR, which was then cloned into the pMir-Report vector (Thermo Fisher Scientific, Inc.), resulting in the generation of pMIR-ZEB2. Mutations were introduced within the seed sequences of the 3'UTR of ZEB2 using the QuikChange Site-Directed Mutagenesis kit (Stratagene; Agilent Technologies, Inc., Santa Clara, CA, USA). The mutated ZEB2 3'UTR was then cloned into the pMir-Report vector, generating pMIR-Mut ZEB2. A549 NSCLC cells were co-transfected with $100 \mathrm{ng}$ pMIR-ZEB2 or pMIR-ZEB2-Mut vector and $100 \mathrm{mM}$ miR-200c mimic or scramble miR mimic, and the pRL-TK plasmid (Promega Corporation, Madison, WI, USA) for internal normalization. Following a $36 \mathrm{~h}$ incubation at $37^{\circ} \mathrm{C}$ in an atmosphere containing $5 \% \mathrm{CO}_{2}$, the cells were lysed using lysis buffer (Promega Corporation), and the Dual-Luciferase Reporter Assay system (Promega Corporation) was used to conduct luciferase reporter gene assay on an LD400 luminometer (Beckman Coulter, Brea, CA, USA), according to the manufacturer's protocol. Data are presented as the ratio of Renilla luciferase to firefly luciferase.

Statistical analysis. Data are presented as the mean \pm standard deviation from at least three separate experiments. SPSS 18.0 software (SPSS, Inc., Chicago, IL, USA) was used for statistical analysis. Differences between the groups were determined using one-way analysis of variance. $\mathrm{P}<0.05$ was considered to indicate a statistically significant difference.

\section{Results}

miR-200c is significantly downregulated in NSCLC cell lines. RT-qPCR was conducted to detect the expression levels of miR-200c in the following NSCLC cell lines: A549, H358, H460 and H1229, and in the BEAS-2B normal human lung epithelial cell line, which was used as a control. The expression levels of miR-200c were significantly reduced in the NSCLC cell lines compared with in the BEAS-2B cells. The A549 cells exhibited the most significant decrease in the expression levels of miR-200c $(\mathrm{P}<0.05$; Fig. 1).

Overexpression of miR-200c suppresses the migration and invasion of NSCLC cells. To investigate the function of miR-200c in NSCLC in vitro, A549 cells were transfected with a miR-200c mimic or a scramble mimic, which was used as negative control. Post-transfection, RT-qPCR was conducted to detect the expression levels of miR-200c. As shown in Fig. 2A, the expression levels of miR-200c were significantly increased in the miR-200c mimic group compared with in the control group $(\mathrm{P}<0.05)$, whereas transfection with the negative control did not affect the expression levels of miR-200c in A549 cells. Subsequently, wound healing and Transwell assays were conducted to determine cell migration and invasion in each group. As shown in Fig. 2B and C, overexpression of miR-200c markedly suppressed A549 cell migration and invasion, compared with in the control group $(\mathrm{P}<0.05)$. These results suggest that miR-200c may exert inhibitory effects on the metastasis of NSCLC cells.

ZEB2 is a direct target of miR-200c in NSCLC cells. Three computational algorithms: PicTar (http://pictar.mdc-berlin. de/), TargetScan (http://www.targetscan.org/), and miRanda (http://www.microrna.org/microrna/home.do), were used to search for potential target genes of miR-200c. Among all putative targets of miR-200c, ZEB2 was evolutionarily conserved, and perfect base pairing was observed between the seed sequence of mature miR-200c and the 3'UTR of ZEB2 mRNA (Fig. 3A). Total cDNA from A549 cells was used to amplify the 3'UTR of ZEB2, which was then cloned into the pMir-Report vector, generating pMIR-ZEB2. In addition, mutations were introduced within the seed sequences of the 3'UTR of ZEB2 (Fig. 4A), and were then cloned into the pMir-Report vector, generating pMIR-Mut ZEB2. Subsequently, a luciferase reporter assay was used to determine whether miR-200c was able to directly bind to seed sequences in the ZEB2 3'UTR in NSCLC A549 cells. As shown in Fig. 3B, the luciferase activity was markedly reduced in A549 cells co-transfected with pMIR-ZEB2 and miR-200c mimic $(\mathrm{P}<0.05)$; however, no difference was detected in the A549 cells co-transfected with pMIR-Mut ZEB2 and miR-200c mimic, as compared with the control group. These findings indicate that ZEB2 is a direct target of miR-200c in NSCLC cells.

The present study also determined the protein expression levels of ZEB2 in A549 cells following upregulation or downregulation of miR-200c. As shown in Fig. 3C, transfection with a miR-200c mimic led to increased expression of miR-200c, whereas transfection with a miR-200c inhibitor led to decreased expression of miR-200c in A549 cells $(\mathrm{P}<0.05)$. Furthermore, overexpression of miR-200c significantly reduced the protein expression levels of ZEB2, 


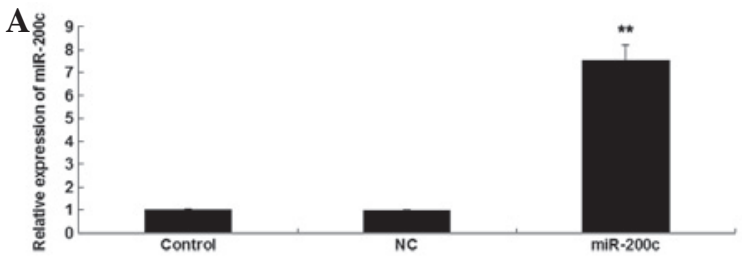

C
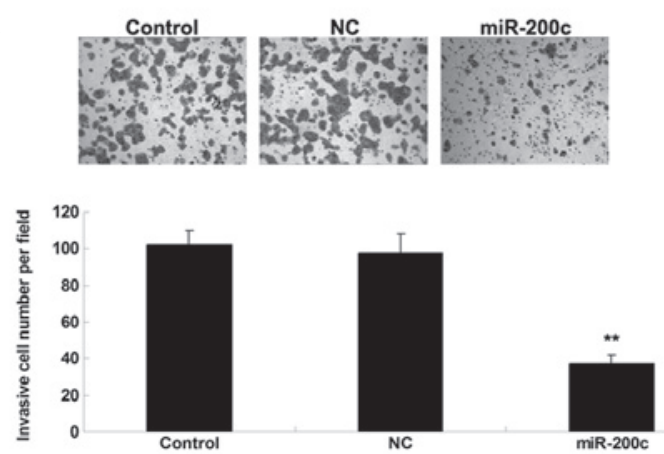

B

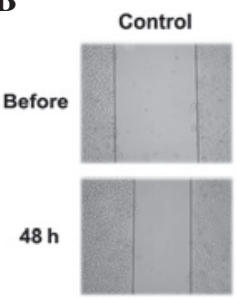

miR-200c
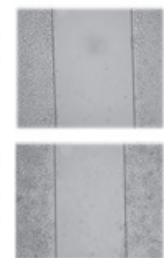

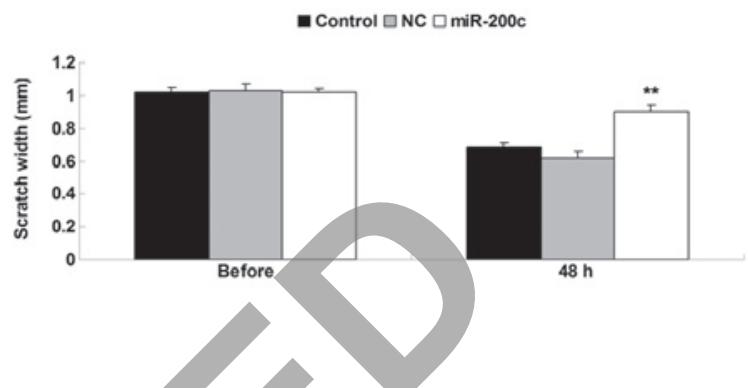

Figure 2. (A) Reverse transcription-quantitative polymerase chain reaction was conducted to detect the expression levels of microRNA (miR)-200c in A549 non-small cell lung cancer cells transfected with a miR-200c mimic or a scramble mimic, which was used as a negative control (NC). (B) Wound healing and (x40 magnification) (C) Transwell assays (x200 magnification) were conducted to determine cell migration and invasion in each group. Non-transfected A549 cells were used as a control. Data are presented as the mean \pm standard deviation. ${ }^{* *} \mathrm{P}<0.05 \mathrm{vs}$. the control cells.

A

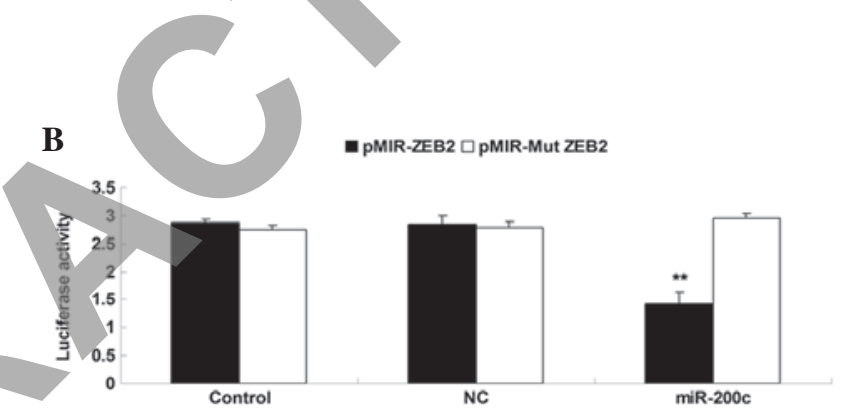

C
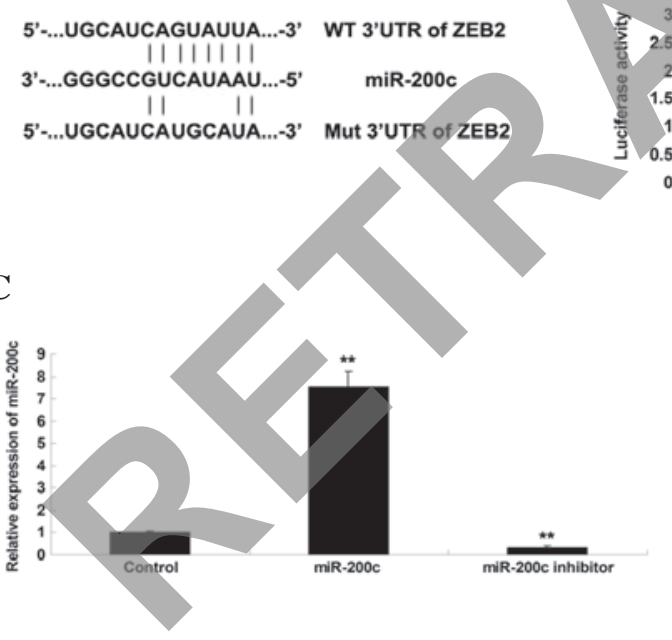

D
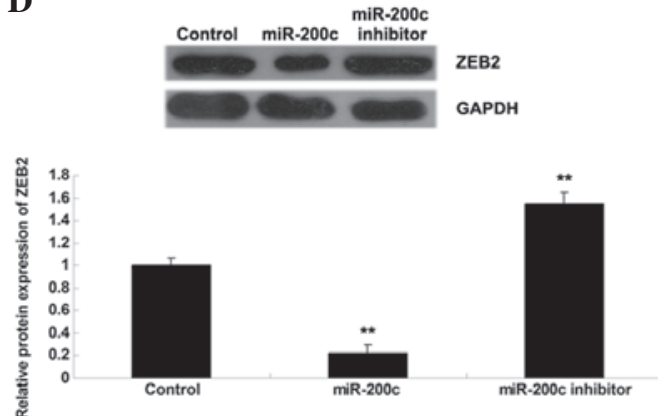

Figure 3. (A) Seed sequences of microRNA (miR)-200c in the wild type (WT) and mutant type (Mut) 3' untranslated region (3'UTR) of zinc finger E-box-binding homeobox 2 (ZEB2) are indicated. (B) Luciferase activity was significantly decreased in A549 cells co-transfected with pMIR-ZEB2 and miR-200c mimics compared with in the control group. However, luciferase activity exhibited no significant alteration in A549 cells co-transfected with pMIR-Mut ZEB2 and miR-200c mimics compared with in the control group. Control group, A549 cells transfected with pMIR-ZEB2 or pMIR-Mut ZEB2 only; A549 negative control (NC) group, cells co-transfected with scramble miR mimic and pMIR-ZEB2 or pMIR-Mut ZEB2. Data are presented as the mean \pm standard deviation. ${ }^{* *} \mathrm{P}<0.01$ vs. the control cells. (C) Reverse transcription-quantitative polymerase chain reaction was conducted to detect the expression levels of miR-200c in A549 non-small cell lung cancer cells transfected with a miR-200c mimic or miR-200c inhibitor. (D) Western blotting was used to examine the protein expression levels of ZEB2. Glyceraldehyde 3-phosphate dehydrogenase (GAPDH) was used as an internal reference. For (C and D) non-transfected A549 cells were used as the control. Data are presented as the mean \pm standard deviation. ${ }^{* *} \mathrm{P}<0.05$ vs. the control cells.

whereas knockdown of miR-200c resulted in a significant increase in ZEB2 protein expression levels in NSCLC A549 cells $(\mathrm{P}<0.05$; Fig. 3D). These findings indicate that miR-200c negatively regulates the protein expression of ZEB2 by directly binding to the 3'UTR of ZEB2 mRNA in A549 NSCLC cells.
Knockdown of ZEB2 inhibits NSCLC cell migration and invasion. Since miR-200c was shown to negatively regulate ZEB2 expression, the present study further investigated whether knockdown of ZEB2 exhibited similar effects on cell migration and invasion in A549 cells, as overexpression of miR-200c. A549 cells were transfected with ZEB2 siRNA, 

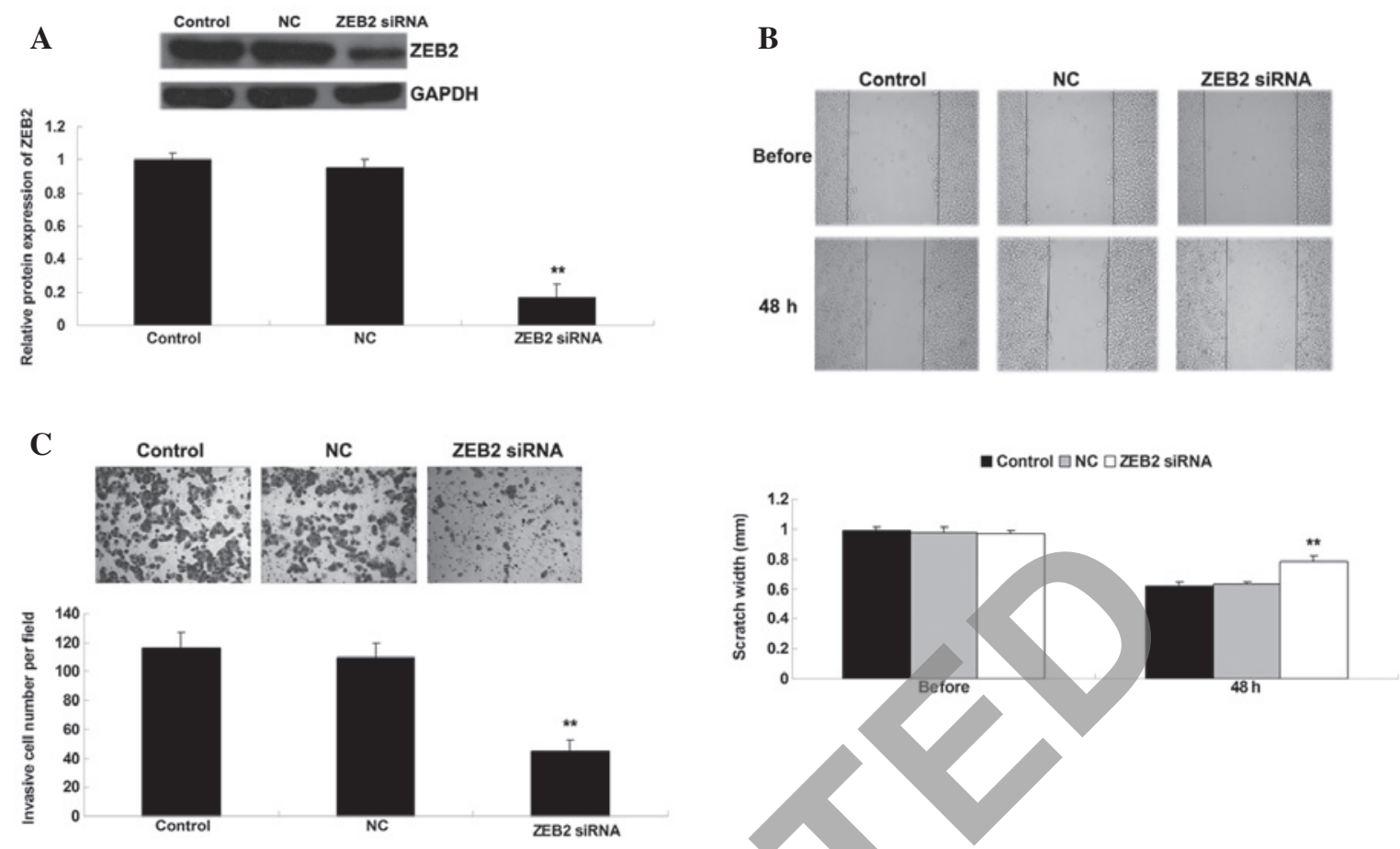

Figure 4. (A) Western blotting was used to examine the protein expression levels of Zinc finger E-box-binding homeobox 2 (ZEB2) in A549 cells transfected with ZEB2 small interfering (si)RNA or negative control (NC) siRNA. Glyceraldehyde 3-phosphate dehydrogenase (GAPDH) was used as an internal reference. (B) Wound healing and (C) Transwell assays were conducted to determine cell migration and invasion in each group. Non-transfected A549 cells were used as a control. Data are presented as the mean \pm standard deviation. ${ }^{* *} \mathrm{P}<0.05$ vs. the control cells.

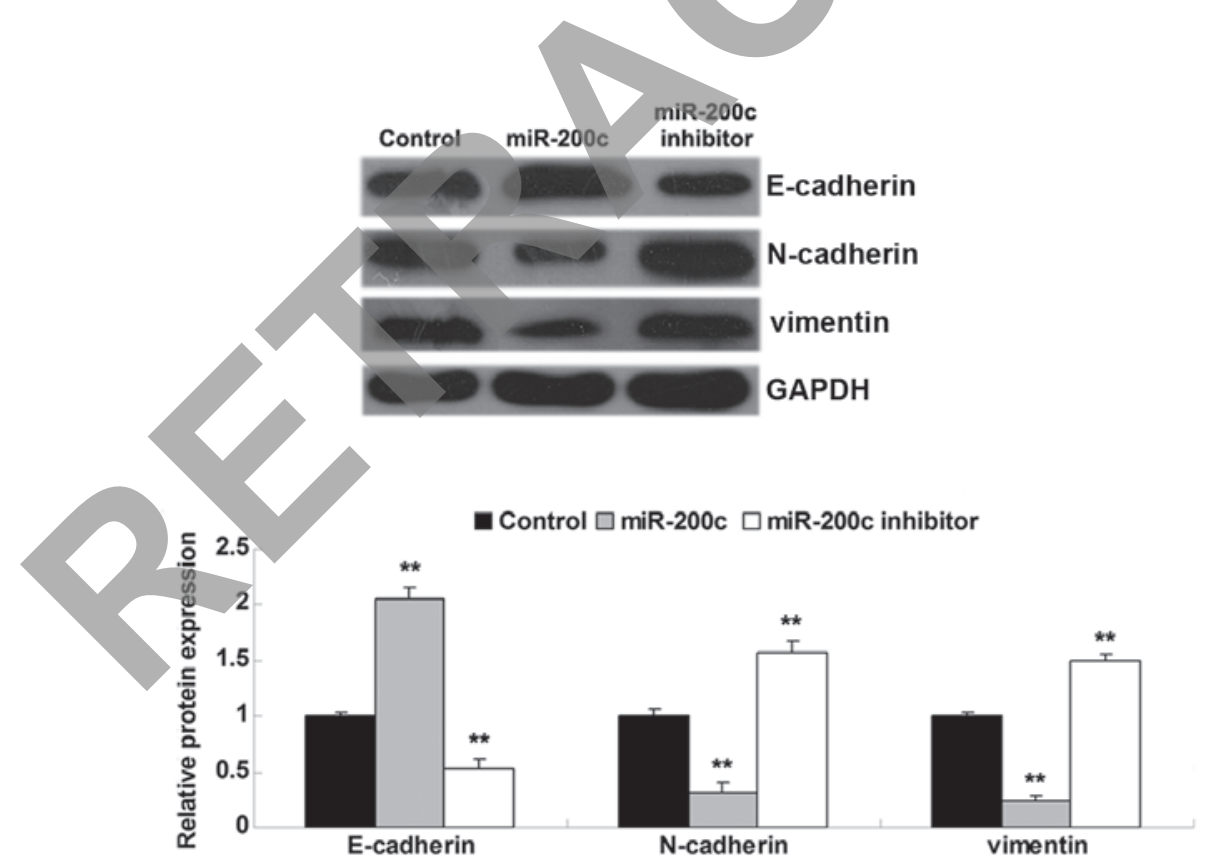

Figure 5. Western blotting was used to examine the protein expression levels of E-cadherin, N-cadherin and vimentin in A549 cells transfected with a microRNA (miR)-200c mimic or miR-200c inhibitor. Glyceraldehyde 3-phosphate dehydrogenase (GAPDH) was used as an internal reference. Non-transfected A549 cells were used as a control. Data are presented as the mean \pm standard deviation. ${ }^{* *} \mathrm{P}<0.05$ vs. the control cells.

and the protein expression levels of ZEB2 were significantly reduced post-transfection $(\mathrm{P}<0.05$; Fig. $4 \mathrm{~A})$. Wound healing and Transwell assays were then conducted to determine cell migration and invasion. As shown in Fig. 4B and C, knockdown of ZEB2 suppressed the migration and invasion of A549 cells compared with the control group $(\mathrm{P}<0.05)$. These results suggest that miR-200c may negatively mediate cell migration and invasion in NSCLC cells, probably via inhibition of ZEB2.
miR-200c has a suppresses role in the regulation of EMT in NSCLC cells. Since ZEB2 is known to act as a regulator of EMT in human cancer, and the results of the present study established a relationship between miR-200c and ZEB2 in A549 cells, it was hypothesized that the suppressive effects of miR-200c on A549 cell migration and invasion may be associated with ZEB-2-mediated EMT. Therefore, the expression levels of the following EMT-related proteins: 
$\mathrm{N}$-cadherin, E-cadherin and vimentin, were detected in A549 cells following overexpression or knockdown of miR-200c. Western blotting indicated that upregulation of miR-200c led to increased protein expression levels of E-cadherin, and decreased protein expression levels of $\mathrm{N}$-cadherin and vimentin in A549 cells, as compared with in the control group $(\mathrm{P}<0.05$; Fig. 5). Conversely, knockdown of miR-200c led to decreased protein expression levels of E-cadherin, and increased protein expression levels of $\mathrm{N}$-cadherin and vimentin compared with in the control group $(\mathrm{P}<0.05$; Fig. 5). These results suggest that miR-200c negatively regulates EMT in NSCLC cells, probably via inhibition of ZEB2.

\section{Discussion}

Previous studies have reported that miRs have an important role in the development and progression of NSCLC. The present study demonstrated that miR-200c was significantly downregulated in NSCLC cell lines compared with in normal lung epithelial cells. Subsequently, the function of miR-200c was investigated in NSCLC in vitro. Overexpression of miR-200c significantly inhibited the migration, invasion and EMT of NSCLC cells, thus suggesting that the decreased expression of miR-200c may contribute to the metastasis of NSCLC.

Numerous studies have explored the role of miR-200c in NSCLC.Ceppi et al (8) investigated the expression of miR-200c in nine different NSCLC cell lines, and detected a strong inverse correlation with invasion. In addition, overexpression of miR-200c in highly invasive/aggressive NSCLC cells led to a loss of the mesenchymal phenotype, as detected by upregulated E-cadherin and downregulated $\mathrm{N}$-cadherin, and inhibited in vitro cell invasion and in vivo metastatic formation (8). These findings are consistent with those of the present study. Furthermore, overexpression of $\mathrm{miR}-200 \mathrm{c}$ restored sensitivity of NCI-H1299 cells to cisplatin and cetuximab (8). Ceppi et al (8) also examined the expression levels of miR-200c in 69 primary NSCLC tissues, and demonstrated that lower miR-200c expression levels were significantly associated with a poor grade of differentiation, a higher propensity for lymph node metastases, and a lower E-cadherin expression. These findings suggested that miR-200c may have an important role in NSCLC metastasis. Conversely, Liu et al (15) reported that miR-200c was frequently upregulated in NSCLC tissues, and high expression of miR-200c was associated with a poor survival in patients with NSCLC. Furthermore, high miR-200c expression was correlated with shorter overall survival in NSCLC adenocarcinoma, but not in squamous cell carcinoma, thus suggesting a dual role for miR-200c in the prognosis of NSCLC, which may be associated with tumor cell origin (16).

The present study identified ZEB2 as a direct target gene of miR-200c, and demonstrated that miR-200c negatively regulates the protein expression of ZEB2 in A549 NSCLC cells. ZEB2 is a member of the deltaEF-1 family of two-handed zinc-finger factors, and acts as a transcriptional suppressor (17). ZEB2 has been demonstrated to be associated with various types of human cancer, including gastric cancer (18), renal cell carcinoma (19), ovarian cancer (20), glioma (21), pancreatic cancer (22), and lung cancer (23). Fang et al (23) demonstrated that overexpression of ZEB2 was associated with poor pathological stage of small cell lung cancer and shorter survival time, whereas knockdown of ZEB2 sensitized cancer cells to chemotherapeutic drugs by increasing drug-induced cell apoptosis and inducing an $\mathrm{S}$ phase cell cycle arrest. These results suggested that ZEB2 acts as an oncogene in lung cancer. In the present study, knockdown of ZEB2 significantly suppressed the migration, invasion and EMT of NSCLC cells, similar effects to those observed following miR-200c overexpression. In addition, ZEB2 has been identified as a direct target of miR-200c in other cancer types. Gregory et al (24) reported that the miR-200 family (miR-200a, miR-200b, miR-200c, miR-141 and miR-429) and miR-205 were able to suppress EMT in invasive breast cancer cell lines with mesenchymal phenotypes by directly targeting ZEB2. Therefore, the present study provides further evidence regarding the relationship between miR-200c and ZEB2 in human cancer.

In conclusion, the present study demonstrated that miR-200c exerts suppressive effects on the migration, invasion and EMT of NSCLC cells, at least partly by targeting ZEB2, thus suggesting that miR-200c acts as a tumor suppressor in NSCLC metastasis. Therefore, miR-200c may be used for the prevention of NSCLC metastasis.

\section{References}

1. DeSantis C, Ma J, Bryan L and Jemal A: Breast cancer statistics, 2013. CA Cancer J Clin 64: 52-62, 2014.

2. Dimou A and Papadimitrakopoulou V: Non-Small cell lung cancer beyond biomarkers: The evolving landscape of clinical trial design. J Pers Med 4: 386-401, 2014.

3. Chouaid C, Crequit P, Borget I and Vergnenegre A: Economic evaluation of first-line and maintenance treatments for advanced non-small cell lung cancer: A systematic review. Clinicoecon Outcomes Res 7: 9-15, 2014.

4. Moss EG: MicroRNAs: Hidden in the genome. Curr Biol 12: R138-R140, 2002.

5. Choi E, Choi E and Hwang KC: MicroRNAs as novel regulators of stem cell fate. World J Stem Cells 5: 172-187, 2013.

6. LujambioA,Calin GA,VillanuevaA,RoperoS,Sánchez-CéspedesM, Blanco D, Montuenga LM, Rossi S, Nicoloso MS, Faller WJ, et al: A microRNA DNA methylation signature for human cancer metastasis. Proc Natl Acad Sci USA 105: 13556-13561, 2008.

7. Calin GA and Croce CM: MicroRNA signatures in human cancers. Nat Rev Cancer 6: 857-866, 2006.

8. Ceppi P, Mudduluru G, Kumarswamy R, Rapa I, Scagliotti GV, Papotti M and Allgayer H: Loss of miR-200c expression induces an aggressive, invasive and chemoresistant phenotype in non-small cell lung cancer. Mol Cancer Res 8: 1207-1216, 2010.

9. Li J, Tan Q, Yan M, Liu L, Lin H, Zhao F, Bao G, Kong H, Ge C, Zhang F, et al: miRNA-200c inhibits invasion and metastasis of human non-small cell lung cancer by directly targeting ubiquitin specific peptidase 25. Mol Cancer 13: 166, 2014.

10. Shi L, Zhang S, Wu H, Zhang L, Dai X, Hu J, Xue J, Liu T, Liang Y and Wu G: MiR-200c increases the radiosensitivity of non-small-cell lung cancer cell line A549 by targeting VEGF-VEGFR2 pathway. PLoS One 8: e78344, 2013.

11. Remacle JE, Kraft H, Lerchner W, Wuytens G, Collart C, Verschueren K, Smith JC and Huylebroeck D: New mode of DNA binding of multi-zinc finger transcription factors: deltaEF1 family members bind with two hands to two target sites. EMBO J 18: 5073-5084, 1999.

12. Nam EH, Lee Y, Park YK, Lee JW and Kim S: ZEB2 upregulates integrin alpha5 expression through cooperation with Spl to induce invasion during epithelial-mesenchymal transition of human cancer cells. Carcinogenesis 33: 563-571, 2012.

13. You J, Li Y, Fang N, Liu B, Zu L, Chang R, Li X and Zhou Q: MiR-132 suppresses the migration and invasion of lung cancer cells via targeting the EMT regulator ZEB2. PLoS One 9: e91827, 2014.

14. Livak KJ and Schmittgen TD: Analysis of relative gene expression data using real-time quantitative PCR and the 2(-Delta Delta C(T)) Method. Methods 25: 402-408, 2001. 
15. Liu XG, Zhu WY, Huang YY, Ma LN, Zhou SQ, Wang YK, Zeng F, Zhou JH and Zhang YK: High expression of serum miR-21 and tumor miR-200c associated with poor prognosis in patients with lung cancer. Med Oncol 29: 618-626, 2012.

16. Tejero R, Navarro A, Campayo M, Viñolas N, Marrades RM, Cordeiro A, Ruíz-Martínez M, Santasusagna S, Molins L, Ramirez J and Monzó M: miR-141 and miR-200c as markers of overall survival in early stage non-small cell lung cancer adenocarcinoma. PLoS One 9: e101899, 2014.

17. Yang X, Wang J, Qu S, Zhang H, Ruan B, Gao Y, Ma B, Wang X, Wu N, Li X, et al: MicroRNA-200a suppresses metastatic potential of side population cells in human hepatocellular carcinoma by decreasing ZEB2. Oncotarget 6: 7918-7929, 2015.

18. Zhou X, Wang Y, Shan B, Han J, Zhu H, Lv Y, Fan X, Sang M, Liu XD and Liu W: The downregulation of miR-200c/141 promotes ZEB1/2 expression and gastric cancer progression. Med Oncol 32: 428, 2015.

19. Chen Z, Tang ZY, He Y, Liu LF, Li DJ and Chen X: miRNA-205 is a candidate tumor suppressor that targets ZEB2 in renal cell carcinoma. Oncol Res Treat 37: 658-664, 2014.
20. Guo F, Cogdell D, Hu L, Yang D, Sood AK, Xue F and Zhang W: MiR-101 suppresses the epithelial-to-mesenchymal transition by targeting ZEB1 and ZEB2 in ovarian carcinoma. Oncol Rep 31: 2021-2028, 2014.

21. Qi S, Song Y, Peng Y, Wang H, Long H, Yu X, Li Z, Fang L, Wu A, Luo W, et al: ZEB2 mediates multiple pathways regulating cell proliferation, migration, invasion and apoptosis in glioma. PLoS One 7: e38842, 2012

22. Usova EV, Kopantseva MR, Kostina MB, Van'kovich AN, Egorov VI and Kopantsev EP: Expression of the ZEB2 gene in pancreatic stromal cells in pancreatic ductal adenocarcinoma, pancreatitis and normal state. Dokl Biol Sci 448: 61-64, 2013.

23. Fang S, Zeng X, Zhu W, Tang R, Chao Y and Guo L: Zinc finger E-box-binding homeobox 2 (ZEB2) regulated by miR-200b contributes to multi-drug resistance of small cell lung cancer. Exp Mol Pathol 96: 438-444, 2014.

24. Gregory PA, Bert AG, Paterson EL, Barry SC, Tsykin A, Farshid G, Vadas MA, Khew-Goodall Y and Goodall GJ: The miR-200 family and miR-205 regulate epithelial to mesenchymal transition by targeting ZEB1 and SIP1. Nat Cell Biol 10: 593-601, 2008. 